\title{
Exosomes from miR-29a-modified adipose-derived mesenchymal stem cells reduce excessive scar formation by inhibiting TGF-ß32/Smad3 signaling
}

\author{
RUIHONG YUAN, XIAOMING DAI, YISONG LI, CHUNSHAN LI and LIU LIU \\ Department of Plastic Surgery, The First Affiliated Hospital of Kunming \\ Medical University, Kunming, Yunnan 650032, P.R. China
}

Received October 5, 2020; Accepted June 2, 2021

DOI: $10.3892 / \mathrm{mmr} .2021 .12398$

\begin{abstract}
Pathological scars mainly refer to hypertrophic scars and keloids, and have a high incidence. Moreover, these scars seriously affect the patient's appearance and are associated with significant pain. The present study aimed to investigate the inhibitory effect of microRNA (miR)-29a from human adipose-derived mesenchymal stem cells (hADSCs) exosomes on scar formation. Firstly, the expression of miR-29a in thermal skin tissues of mice and human hypertrophic scar fibroblasts (HSFBs) was detected via reverse transcription-quantitative PCR. Exosomes derived from miR-29a-modified hADSCs were extracted and the influence of miR-29a-modified hADSCs-exo on the proliferation and function of HSFBs was determined. Lastly, the effect of miR-29a-modified hADSCs-exo on scar formation was determined using a thermal mouse model. The results demonstrated that miR-29a was downregulated in scar tissues after scalding and in HSFBs. After treating HSFBs with miR-29a-modified hADSC exosomes, miR-29a-overexpressing hADSC exosomes inhibited the proliferation and migration of HSFBs. Moreover, it was found that TGF- $\beta 2$ was the target of miR-29a, and that hADSC exosome-derived miR-29a inhibited the fibrosis of HSFBs and scar hyperplasia after scalding in mice by targeting the TGF- $\beta 2 / \mathrm{Smad} 3$ signaling pathway. In summary, the current data indicated that miR-29a-modified hADSC exosome therapy can decrease scar formation by inhibiting the TGF- $\beta 2 /$ Smad3 signaling pathway via its derived exogenous miR-29a, and this may be useful for the future treatment of pathological scars by providing a potential molecular basis.
\end{abstract}

Correspondence to: Dr Liu Liu, Department of Plastic Surgery, The First Affiliated Hospital of Kunming Medical University, 295 Xichang Road, Xishan, Kunming, Yunnan 650032, P.R. China E-mail: liuliu1234562020@163.com

Key words: pathological scar, adipose-derived mesenchymal stem cells, exosomes, microRNA-29a, TGF- $\beta 2$

\section{Introduction}

Operations, traumas, burns and scalds can cause skin trauma, and the rapid and effective repair of skin wounds is key to ensuring the function of the skin barrier. The process of skin wound healing is a complex regenerative reaction that involves four stages: Hemostasis, inflammatory response, cell proliferation and tissue reconstruction (1). During this dynamic process, excessive collagen deposition and fibroblast proliferation and activation lead to the formation of pathological scars, including hypertrophic scars and keloids (2). Pathological scars cause not only pain and itching but also aesthetic issues and seriously affect the patients' mental state and quality of life. Therefore, studying the mechanism of pathological scar formation and hyperplasia, as well as its prevention and treatment measures is one of the hot topics in the field of medical plastic surgery and has important clinical significance $(3,4)$.

In recent years, stem cell therapy has become an effective treatment and research hotspot of skin wound healing. Previous studies have shown that adipose-derived mesenchymal stem cells (ADSCs), one of the most widely used adult stem cells, have a beneficial ability to promote skin wound healing and reduce scar formation (5-7). Moreover, it has been reported that paracrine cytokines, exosomes and other acellular bioactive derivatives are the main factors via which ADSCs exert their biological functions (8). Exosomes are a bilayer phospholipid membrane structure secreted by cells with a diameter of $40-150 \mathrm{~nm}$ and a density range of $1.09-1.18 \mathrm{~g} / \mathrm{ml}$, and contain proteins, lipids and RNA [mRNA and microRNA (miRNA/miR)] vesicle-like substances (9-11). Exosomes, as important components in the paracrine pathway of stem cells, have high application prospects in promoting the repair and regeneration of skin wounds (12). Compared with the direct use of stem cells for tissue repair, exosomes have improved safety and easier storage and transportation, are fast and efficient, have no ethical restrictions and have a wide range of sources, amongst other advantages $(13,14)$. Previous research has shown that exosomes can participate in various processes of skin tissue repair and regeneration, as well as promote skin healing and skin tissue regeneration by enhancing the proliferation and migration of skin cells $(15,16)$ and angiogenesis $(17,18)$, in addition to regulating the immune 
response (19). Thus, these mechanisms provide a new method to achieve cell-free therapy.

miRNAs are a type of non-coding RNA with a length of 21-23 nucleotides that can affect multiple gene networks simultaneously to coordinate biological responses (20). miRNAs exist in tissues throughout the body and can inhibit the expression of target genes by binding to the 3 ' untranslated region (3'UTR), coding region or 5'UTR of target mRNA, thereby blocking mRNA translation or degrading mRNA (21). Subsequently, miRNAs can participate in cell proliferation and various growth and development processes, such as differentiation, migration, metabolism and apoptosis (22).

Studies have reported that miRNAs have a significant effect in regenerative medicine and can regulate the growth of a variety of tissues in various processes, including skin healing (23), bone regeneration (24), liver regeneration (25), kidney regeneration (26), and myocardial regeneration (27). miRNAs can obviously regulate the proliferation of fibroblasts and the synthesis of extracellular matrix via several molecular mechanisms. Additionally, they are key regulators of skin morphogenesis and wound healing. For example, miR-29a has been reported to inhibit fibroblast proliferation, migration and collagen deposition after skin thermal injury, and can promote the repair of denatured dermis $(28,29)$. Furthermore, mesenchymal stem cell (MSC) therapy can improve skin wound healing in diabetic mice by correcting the miR-29a imbalance (30). A recent study revealed that the expression level of miR-29a was significantly downregulated in human skin keloids, and long non-coding RNA H19 promoted the proliferation and metastasis of fibroblasts by modifying downstream miR-29a and collagen type I $\alpha 1$ chain (31), suggesting that the downregulation of miR-29a is closely associated with the formation of keloids induced by excessive proliferation of fibroblasts.

The TGF- $\beta /$ Smad signaling pathway is considered an important regulator during skin wound healing and pathological scar formation (32). Studies have shown that miR-29b (another member of the miR-29a family) can target TGF- $\beta 1$ in fibroblasts to regulate the activation of the TGF- $\beta 1 / \mathrm{Smad} 3$ signaling pathway, and thus, promote skin wound healing and reduce excessive scar formation $(6,33,34)$. miR-29a has also been reported to attenuate Angiotensin II-induced left ventricular remodeling by inhibiting TGF- $\beta /$ Smad signaling pathway (35). However, whether miR-29a reduces excessive scar formation via TGF- $\beta /$ Smad signaling pathway remains unknown.

Previous studies have reported that miR-29a was stably expressed in the exosomes of human ADSCs (hADSCs-exo) (36), but whether ADSCs-exo can inhibit scar formation by delivering exogenous miR-29a to the wound site is yet to be determined. The present study aimed to evaluate the inhibitory effect of hADSCs-exo enriched with miR-29a on scar formation for wound healing purposes.

\section{Materials and methods}

Cell culture and transfection. All cells were purchased from BeNa Culture Collection. hADSCs (cat. no. BNCC340147), human skin fibroblasts (HSFs; cat. no. BNCC353686) and human hypertrophic scar fibroblasts (HSFBs; cat. no. BNCC342248) were cultured in DMEM (HyClone; Cytiva) containing 10\% FBS (Gibco; Thermo Fisher Scientific, Inc.) and $1 \%(\mathrm{v} / \mathrm{v})$ penicillin/streptomycin (Gibco; Thermo Fisher Scientific, Inc.) and were then incubated at $37^{\circ} \mathrm{C}$ in a humidified atmosphere containing $5 \% \mathrm{CO}_{2}$. All cell experiments were approved by the Ethics Committee of The First Affiliated Hospital of Kunming Medical University (approval no. kmmu2021188) due to the use of non-immortalized human cells, and carried out in accordance with the cell experiment management regulations of the hospital.

For the transfection experiment, miR-29a mimics (5'-UAG CACCAUCUGAAAUCGGUUA-3'), miR-29a inhibitor (5'-UAACCGAUUUCAGAUGGUGCUA-3') and corresponding negative controls (NC; mimics-NC: 5'-UUCUCCG AACGUGUCACGU-3'; inhibitor-NC: 5'-CAGUACUUUUGU GUAGUACAA-3') were constructed by Guangzhou RiboBio Co., Ltd. hADSCs were seeded into 6-well plates at $1.5 \times 10^{5}$ cells/well for $24 \mathrm{~h}$. Then, $100 \mathrm{nM}$ miR-29a mimics or miR-29a inhibitor was transfected intohADSCs withLipofectamine ${ }^{\circledR} 2000$ transfection reagent (Invitrogen; Thermo Fisher Scientific, Inc.), following the manufacturer's instructions. Transfection was performed for $6 \mathrm{~h}$ at $37^{\circ} \mathrm{C}$ with $5 \% \mathrm{CO}_{2}$. At $4 \mathrm{~h}$ post-transfection, the transfection efficiency was detected via reverse transcription-quantitative (RT-q)PCR (Fig. S1).

Exosome extraction and identification. Subconfluent hADSCs in the log phase, which were obtained by filtering the supernatants through $0.22 \mu \mathrm{m}$ pore filters (MilliporeSigma) and ultracentrifuging the serum at $100,000 \times \mathrm{g}\left(4^{\circ} \mathrm{C}\right.$ for $\left.70 \mathrm{~min}\right)$, were supplemented with $10 \%$ exosome-free FBS. After $24 \mathrm{~h}$ of incubation at $37^{\circ} \mathrm{C}$, conditioned medium was collected for exosome extraction. Exosomes derived from hADSCs (with or without miR-29a mimics and inhibitor transfection) were extracted and purified using a Cell Culture Media Exosome Purification Mini kit (Norgen Biotek Corp.), according to the manufacturer's instructions. To observe the ultrastructure of exosomes, $10 \mu \mathrm{l}$ exosome samples were added to the copper mesh for precipitation for $1 \mathrm{~min}$, and then the floating solution was removed by filter paper. Subsequently, $10 \mu$ uranyl acetate was added to the copper mesh to precipitate for $1 \mathrm{~min}$, and then the floating solution was removed using filter paper. After drying at room temperature for several minutes, the image was detected by transmission electron microscopy (Libra 120; Zeiss AG) at $100 \mathrm{kV}$. The size distribution and concentration of exosomes were determined via nanoflow cytometry (N30 NanoAnalyzer; NanoFCM, Inc.).Antibodies against exosomes susceptibility (TSG)101 (1:1,000; cat. no. ab125011; Abcam), CD63 (1:1,000; cat. no. ab134045; Abcam) and CD81 (1:1,000; cat. no. ab109201; Abcam), which are representative markers of exosomes, and the cellular protein actin (1:5,000; cat. no. ab6276; Abcam) were used to identify the collected exosomes via western blotting or nanoflow cytometry.

Effect of hADSCs-exo on the proliferation of HSFBs. HSFBs were precultured in 6-well plates $\left(1 \times 10^{4}\right.$ cells/well) in DMEM containing $10 \% \mathrm{FBS}$ and $1 \%(\mathrm{v} / \mathrm{v})$ penicillin/streptomycin for $4 \mathrm{~h}$. Then, $20 \mu \mathrm{g}$ miR-29a-modified hADSCs-exo (mimics-exo or inhibitor-exo) was added to the culture medium of HSFBs. An equivalent volume of exosome diluent PBS was added as the control group. After the exosomes were added, the cells 
were incubated at $37^{\circ} \mathrm{C}$ with $5 \% \mathrm{CO}_{2}$ and imaged at 0,24 and $48 \mathrm{~h}$ using an inverted-phase contrast light microscope (Olympus Corporation; magnification, x10) to observe the proliferation of the cells.

In addition, whether HSFBs can directly take up in hADSCs-exo was detected via immunofluorescence labeling. Exosomes were labeled with the red fluorescent linker PKH67 (Sigma-Aldrich; Merck KGaA), as previously reported (37). HSFBs were treated with PKH67-labeled exosomes for $24 \mathrm{~h}$ at $37^{\circ} \mathrm{C}$ with $5 \% \mathrm{CO}_{2}$. DAPI dye (cat. no. ab104139; Abcam) was used for nuclear staining for $10 \mathrm{~min}$ at room temperature. After washing by distilled water to remove the uninternalized exosomes and excess DAPI dye, the fluorescent images were visualized using a confocal microscope (Olympus Corporation; magnification, $\mathrm{x} 40$ ).

Scratch wound assay. As previously described, an in vitro scratch wound assay was performed to evaluate cell migration (38). Briefly, HSFBs were cultured in 6-well culture plates ( $1 \times 10^{4}$ cells/well) along with DMEM containing 10\% FBS until $100 \%$ confluency, and then a scratch was created in the middle of the culture dish on each well using a 200- $\mu$ l pipette tip (Pipet Tip Finder, LLC). The wound was $0.45-0.50 \mathrm{~mm}$ in width per well. Next, the cultures were switched to serum-free medium, and $20 \mu \mathrm{g}$ miR-29a-modified hADSCs-exo (mimics-exo or inhibitor-exo) was added to the culture medium for 24 and $48 \mathrm{~h}$. Digital images of each wound were acquired under an inverted-phase contrast microscope (Olympus Corporation; magnification, x10) at 0,24 and $48 \mathrm{~h}$ after scratching. Wound closure (cell migration) was investigated by measuring the wound area using the commercial software ImageJ (version 1.52a; National Institutes of Health). The results are presented as the percentage of the initial wound area using the following formula: Scratch healing rate $(\%)=$ $\left(\right.$ Area $_{24 \mathrm{~h}}\left(\mathrm{o}\right.$ Area $\left._{48 \mathrm{~h}}\right)-$ Area $\left._{0 \mathrm{~h}}\right) /$ Area $_{0 \mathrm{~h}} \times 100 \%$. Images of each wound were acquired in three random views, and the mean cell migration rate of each sample \pm SD was presented as the final result. The assay was repeated in three pooled cell samples.

Dual-luciferase reporter assay. The binding sites between miR-29a and TGF- $\beta 2$ were predicted with StarBase (version 2.0; http://starbase.sysu.edu.cn/agoClipRNA. php? source $=$ mRNA). Luciferase vectors containing the 3'UTR of human TGF- $\beta 2$ with miR-29a binding sites and mutant miR-29a binding sites were purchased from Shanghai GenePharma Co., Ltd. Then, 50 ng vectors were co-transfected with $20 \mathrm{nM}$ miR-29a mimics or mimics-NC into $1 \times 10^{4}$ HSFB cells using Lipofectamine ${ }^{\circledR} 2000$ transfection reagent (Invitrogen; Thermo Fisher Scientific, Inc.) for $6 \mathrm{~h}$ in the cell incubator with $5 \% \mathrm{CO}_{2}$ at $37^{\circ} \mathrm{C}$. The pRL-CMV vector containing the CMV enhancer and early promoter elements of Renilla luciferase (Promega Corporation) was used as an internal control. After $48 \mathrm{~h}$, the luciferase reporter activity was determined using a Dual-Luciferase ${ }^{\circledR}$ Reporter Assay system (Promega Corporation). Firefly luciferase activity was normalized to Renilla luciferase activity.

TGF- $\beta 2 /$ Smad 3 agonist and TGF- $\beta$ signaling inhibitor treatment. To verify whether miR-29a modified hADSCs-exo altered the fibrosis of HSFBs via TGF- $\beta 2 /$ Smad3 signaling, the TGF- $\beta 2 /$ Smad3 agonist SRI-011381 hydrochloride
(10 $\mu \mathrm{M}$; cat. no. HY-100347A; MedChemExpress) was used to co-treat HSFBs with mimics-exo, and the TGF- $\beta$ signaling inhibitor pirfenidone (AMR69; $3 \mathrm{mM}$; cat. no. HY-B0673; MedChemExpress) was used to co-treat HSFBs with inhibitor-exo. Then the cells were incubated at $37^{\circ} \mathrm{C}$ with $5 \% \mathrm{CO}_{2}$ for $24 \mathrm{~h}$ prior to analysis via western blotting.

Animals and experimental protocol. To investigate the effect of miR-29a-modified hADSCs-exo on skin wound healing and scar formation, a scald skin model was established in mice according to a previous report (33). A total of 36 adult male Kunming mice (age, 6-8 weeks; weight, $22 \pm 2 \mathrm{~g}$ ) from the same generation were purchased from Hunan SJA Laboratory Animal Co., Ltd. (certificate no. 43004700043639). The mice were randomly divided into four groups: Control group $(n=12)$, thermal injury group (thermal; $n=12$ ), exosomes from miR-29a overexpressed hADSCs treated group (mimics-exo; $n=6$ ) and mimics-exo co-treated with the TGF- $\beta$ agonist SRI-011381 hydrochloride group (mimics-exo + SRI-011381; $\mathrm{n}=6$ ). Mice were housed individually with a 12 -h light/dark cycle at $22^{\circ} \mathrm{C}$ with $50 \%$ humidity and received ad libitum food and water. All experiments were approved by the Animal Ethics Committee of the First Affiliated Hospital of Kunming Medical University (approval no. kmmu2021188).

Before thermal injury, the mice were anesthetized with an intraperitoneal injection of $1 \%$ sodium pentobarbital $(40 \mathrm{mg} / \mathrm{kg})$ and shaved to expose the dorsal skin. After disinfecting the back skin with $75 \%$ alcohol, a plastic tube with a diameter of $2 \mathrm{~cm}$ was placed on the exposed back skin, one end of which was tightly connected with the skin. Boiling water at $100^{\circ} \mathrm{C}$ was injected from the other end, and the tube and boiling water were removed after continuous contact with the skin for $10 \mathrm{sec}$. After thermal injury, the mice were housed individually, the mimics-exo and mimics-exo + SRI-011381 groups were multidirectionally, subcutaneously injected with $200 \mu \mathrm{g}$ exosomes from miR-29a-overexpressing hADSCs, and the mimics-exo + SRI-011381 group was intraperitoneally injected with SRI-011381 (RayBiotech, Inc.) at a dosage of $30 \mathrm{mg} / \mathrm{kg}$ at the same time. Meanwhile, the control and thermal injury groups were subcutaneously injected with PBS at the same volume. All mice were treated once every 3 days, and 15 days after thermal injury, all mice were euthanized by an intraperitoneal injection of excessive $1 \%$ sodium pentobarbital $(120 \mathrm{mg} / \mathrm{kg})$, and scalded skin tissues were collected for further tests.

RT-qPCR analysis. RT-qPCR analysis was performed on an ABI 7500 Real-Time PCR system (Applied Biosystems; Thermo Fisher Scientific, Inc.). All reactions were run in triplicate. The thermocycling conditions used for qPCR were as follows: Initial denaturation at $95^{\circ} \mathrm{C}$ for $10 \mathrm{sec}$; followed by $40 \mathrm{cycles}$ of $95^{\circ} \mathrm{C}$ for $10 \mathrm{sec}$ and $60^{\circ} \mathrm{C}$ for $30 \mathrm{sec}$. The $2^{-\Delta \Delta \mathrm{Cq}}$ method was used to calculate relative expression levels (39), and U6 was used to normalize the miRNA expression. Total RNA was extracted from cells or tissues using TRIzol ${ }^{\circledR}$ reagent (Invitrogen; Thermo Fisher Scientific, Inc.), and miRNA RT-qPCR was carried out using a TaqMan ${ }^{\mathrm{TM}}$ MicroRNA Reverse Transcription kit (Applied Biosystems; Thermo Fisher Scientific, Inc.) and TaqMan Universal PCR Master mix (Applied Biosystems; Thermo Fisher Scientific,Inc.) according to the manufacturer's instructions. The primer 

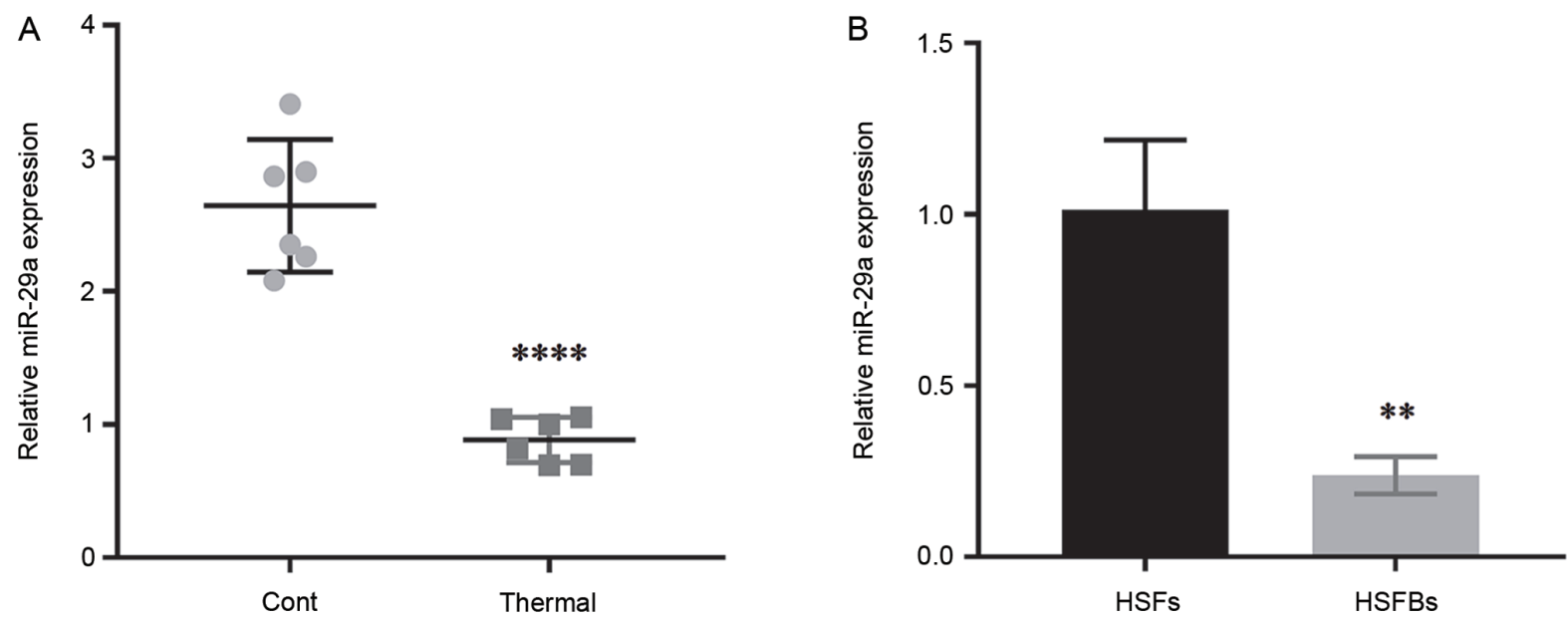

Figure 1. Expression level of miR-29a in scar tissues and HSFBs. (A) Relative expression level of miR-29a in normal skin tissues (Cont) and thermally injured tissues in mice. ${ }^{* * * *} \mathrm{P}<0.0001$ vs. Cont. (B) Relative expression level of miR-29a in HSFs and in HSFBs. Unpaired Student's t-test was used to compare the difference between two groups: ${ }^{* *} \mathrm{P}<0.01$ vs. HSFs. HSFBs, human hypertrophic scar fibroblasts; HSFs, human skin fibroblasts; Cont, control; miR, microRNA.

sequences used were as follows: miR-29a forward, 5'-CTAGCA CCATCTGAAATCGGTTA-3' and reverse, 5'-TGATTGGCT AAAGTCTACCAC-3'; and U6 forward, 5'-CTCGCTTCGG CAGCACATATACT-3' and reverse, 5'-ACGCTTCACGAA TTTGCGTGTC-3'.

Western blotting. The cells or tissues in each group were collected and washed with PBS and RIPA protein lysis buffer (Invitrogen; Thermo Fisher Scientific, Inc.) to extract the total proteins. A BCA protein kit (Pierce; Thermo Fisher Scientific, Inc.) was used to detect the protein concentration and purity. In total, $40 \mu \mathrm{g}$ protein was separated by $10 \%$ SDS-PAGE and then transferred to PVDF membranes via the electric transfer method. The membranes were blocked with a solution containing 5\% skimmed milk powder for $1 \mathrm{~h}$ at room temperature, after which diluted primary antibody was added and the membranes were incubated at $4^{\circ} \mathrm{C}$ overnight. The membranes were then washed three times with membrane washing buffer, and goat anti-rabbit HRP-labeled secondary antibody (1:2,000; cat. no. ab205718; Abcam) or goat anti-mouse HRP-labeled secondary antibody (1:2,000; cat. no. ab205719; Abcam) was added. The blots were incubated for $1 \mathrm{~h}$ at room temperature in the dark and then washed three times with membrane washing buffer. Finally, ECL solution (Bio-Rad Laboratories, Inc.) was added for color development, and images were collected in a gel imaging system. The grayscale value analysis was performed using ImageJ software (version 1.52a; National Institutes of Health), and GAPDH was used as the loading control. The primary antibodies used were as follows: CD63 (1:1,000; cat. no. ab134045; Abcam), CD81 (1:1,000; cat. no. ab109201; Abcam), TSG101 (1:1,000; cat. no. ab125011; Abcam), actin (1:5,000; cat. no. ab6276; Abcam), TGF- $\beta 2$ (1 $\mu \mathrm{g} / \mathrm{ml}$; cat. no. ab113670; Abcam), phosphorylated (p)-Smad3 (1:2,000; cat. no. ab52903; Abcam), $\alpha$-smooth muscle actin ( $\alpha$-SMA; 1:10,000; cat. no. ab124964; Abcam), collagen I (Col-I; 1:1,000; cat. no. ab34710; Abcam), Col-III (1:1,000; cat. no. ab7778; Abcam) and GAPDH (1:1,000; cat. no. ab8245; Abcam).

Histological examinations. Scar tissue samples were fixed in $4 \%$ paraformaldehyde for $24 \mathrm{~h}$ at $4^{\circ} \mathrm{C}$ and then dehydrated by washing with a series of ethanol solutions (50\% ethanol for $2 \mathrm{~h}$; $70 \%$ ethanol for $2 \mathrm{~h}$; $80 \%$ ethanol for $2 \mathrm{~h}$; $95 \%$ ethanol I for $2 \mathrm{~h}$; $95 \%$ ethanol II for $1.5 \mathrm{~h}$; $100 \%$ ethanol I for $1 \mathrm{~h} ; 100 \%$ ethanol II for $30 \mathrm{~min}$ ) at room temperature. Subsequently, samples were embedded in paraffin wax and cut into 5- $\mu \mathrm{m}$ sections for routine $\mathrm{H} \& \mathrm{E}$ and Masson staining. H\&E staining was performed by staining with hematoxylin dye (10 min; room temperature) and eosin dye ( $1 \mathrm{~min}$; room temperature) to observe the morphological changes of scar tissue. All slices were imaged using an inverted-phase contrast light microscope (Olympus Corporation; magnification, $\mathrm{x} 4$ and $\mathrm{x} 20$ ). Scar tissue fibrosis was detected by Masson staining using hematoxylin dye (10 min; room temperature), masson dye (10 min; room temperature) and $1 \%$ light green aqueous solution ( $5 \mathrm{~min}$; room temperature). ImageJ software (version 1.52a; National Institutes of Health) was used to measure the epidermal thickness and Masson-positive area.

Statistical analysis. Data are presented as the mean \pm SEM. The results from the experiments were analyzed using SPSS 20.0 (IBM Corp.). The comparison of multiple groups and the comparison between the two groups were performed by one-way ANOVA or two-way ANOVA followed by Bonferroni post hoc test. A mixed two-way ANOVA followed by Bonferroni post hoc test was also used where appliable. GraphPad Prism 7 (GraphPad Software, Inc.) was used to plot the experimental data. $\mathrm{P}<0.05$ was considered to indicate a statistically significant difference, and all experiments were repeated three times.

\section{Results}

miR-29a expression in mouse scar tissues and HSFBs. To determine the effect of miR-29a on scar formation, the expression level of miR-29a was detected in scar tissues from thermally injured mice and HSFBs, with normal mouse skin tissues and HSFs used as controls. The results demonstrated that the expression level of miR-29a in mouse scar tissues was significantly downregulated compared with that in normal skin tissues (Fig. 1A), and it was also lower in HSFBs (Fig. 1B). These results indicated that miR-29a may act as an inhibitor in the formation of hypertrophic scars. 
A

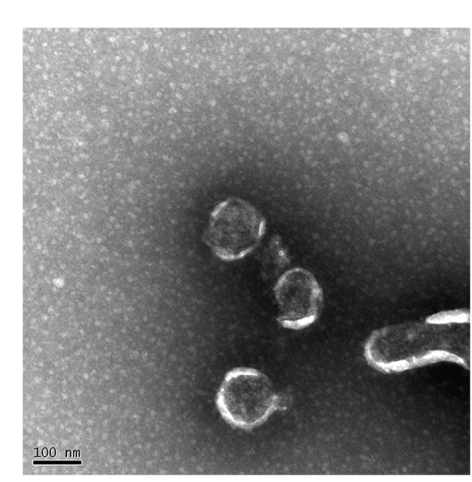

C
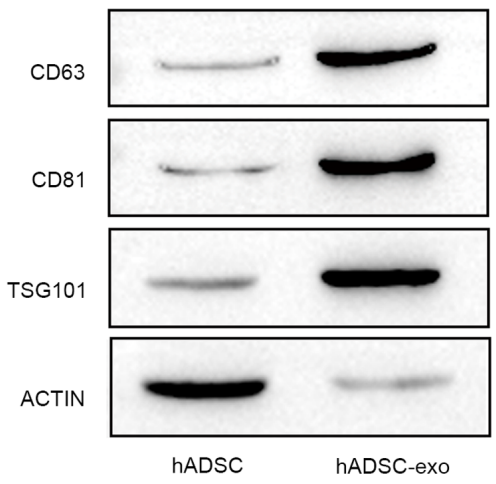

E

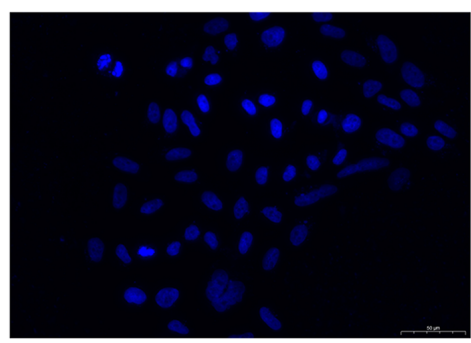

DAPI

F

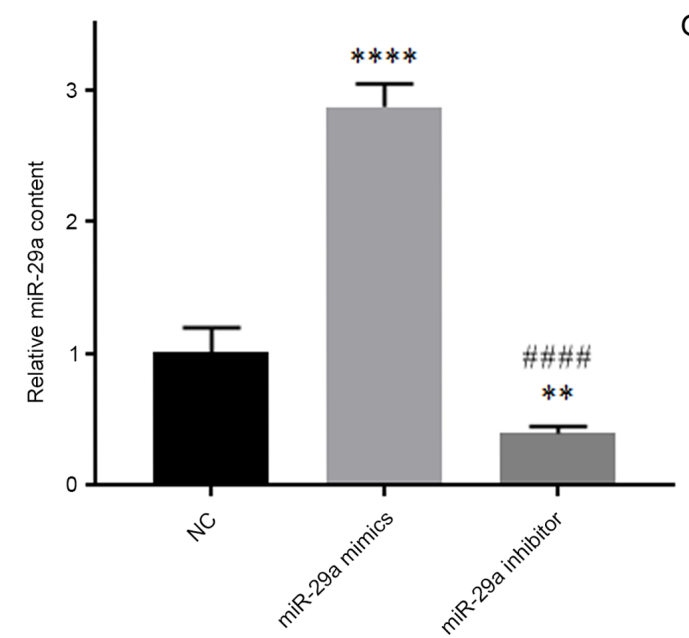

B
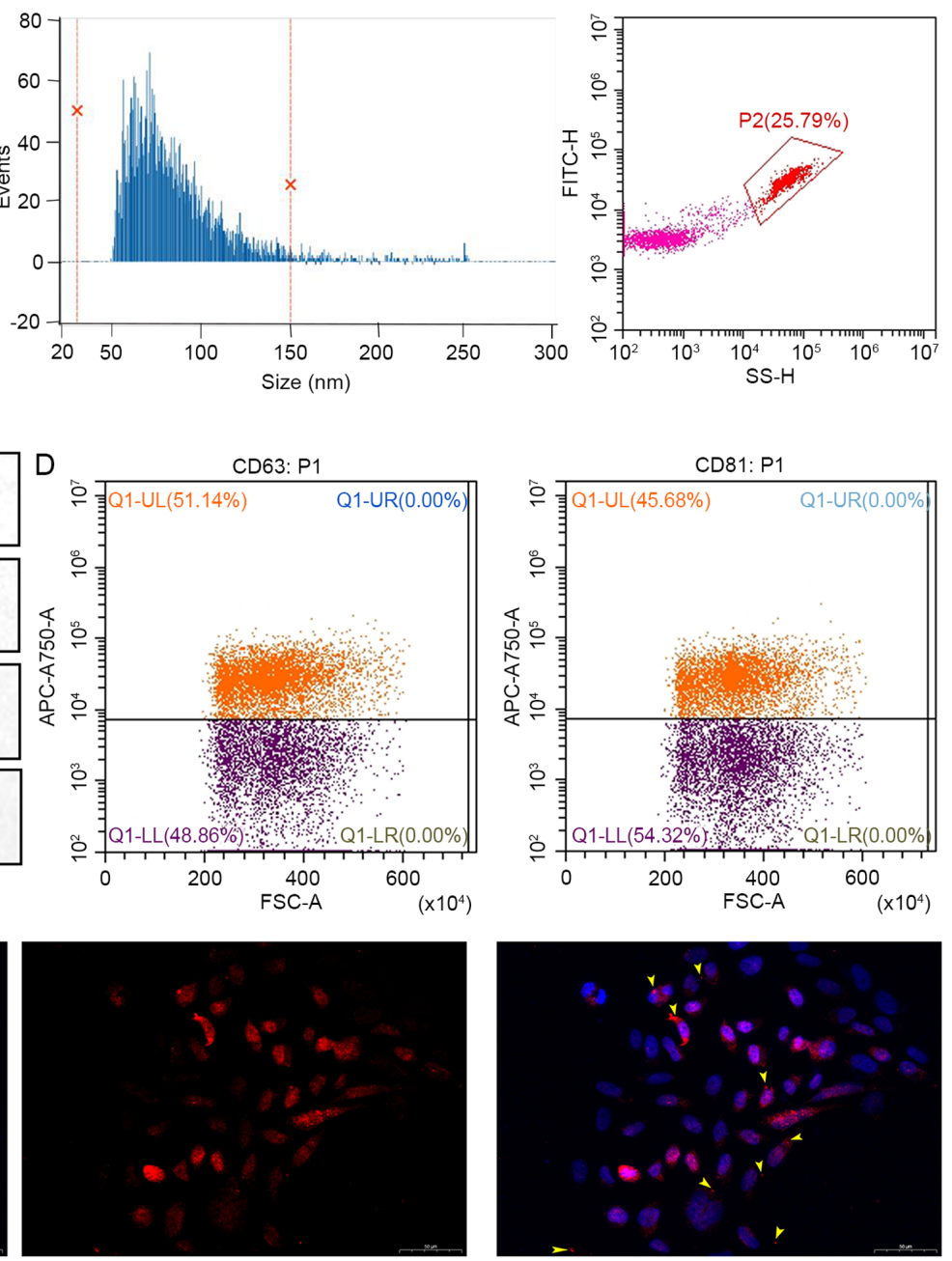

PKH67 exosomes

G

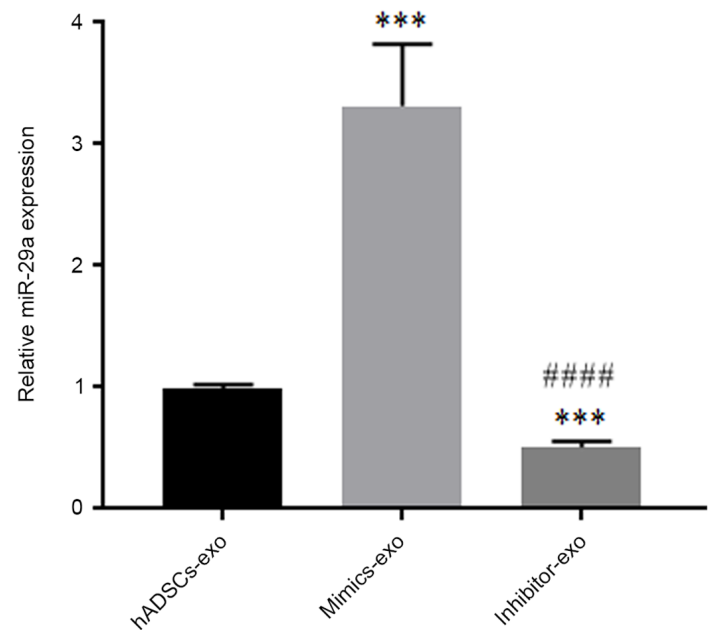

Figure 2. Exosomes extracted from hADSCs and their effect on the expression level of miR-29a in HSFBs. (A) Transmission electron microscopy analysis of exosome ultrastructure. Scale bar, $100 \mathrm{~nm}$. (B) Nanoflow cytometry analysis of particle size distribution and particle number. (C) Western blotting detection of exosome biomarkers. (D) Flow cytometric analysis of the expression levels of CD63 and CD81 in exosomes. (E) Representative images of immunofluorescence labeling of exosomes in HSFBs. Scale bar, $50 \mu \mathrm{m}$. (F) Content of miR-29a in different groups of hADSCs-exo, as analyzed by one-way ANOVA followed by Bonferroni post hoc test. ${ }^{* *} \mathrm{P}<0.01,{ }^{* * * * *} \mathrm{P}<0.0001$ vs. NC group; ${ }^{\# \# \#} \mathrm{P}<0.0001$ vs. miR-29a mimics group. $(\mathrm{G})$ The influence of miR-29a-modified hADSCs-exo on the expression level of miR-29a in HSFBs, as analyzed by one-way ANOVA followed by Bonferroni post hoc test. ${ }^{* * *} \mathrm{P}<0.001$ vs. hADSCs-exo group; ${ }^{\# \# \# ~ P<0.0001 ~ v s . ~ m i m i c s-e x o ~}$ group. NC, negative control; mimics-exo, exosomes from miR-29a overexpressed hADSCs treated group; inhibitor-exo, exosomes from miR-29a knockdown hADSCs treated group; miR, microRNA; hADSCs, human adipose-derived mesenchymal stem cells; TSG101, tumor susceptibility 101.

Extraction of hADSCs-exo and its effect on miR-29a expression in HSFBs. First, exosomes were extracted and purified from hADSCs, then TEM was used to identify the morphology of exosomes extracted from hADSC cultures (Fig. 2A). The 
A

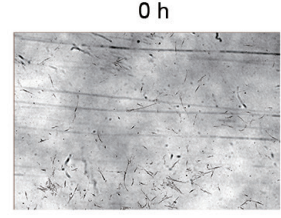

Mimics-exo

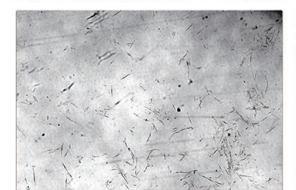

nhibitor-exo

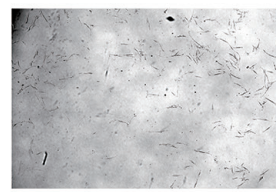

B

NC

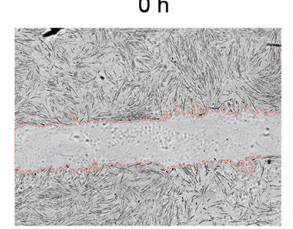

Mimics-exo
$24 \mathrm{~h}$
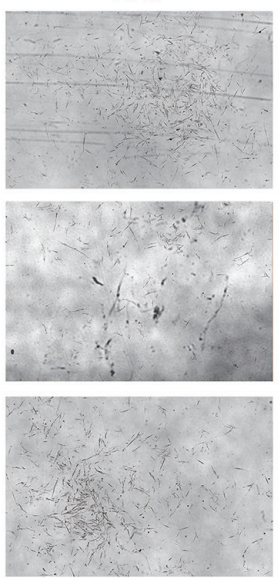

$24 \mathrm{~h}$
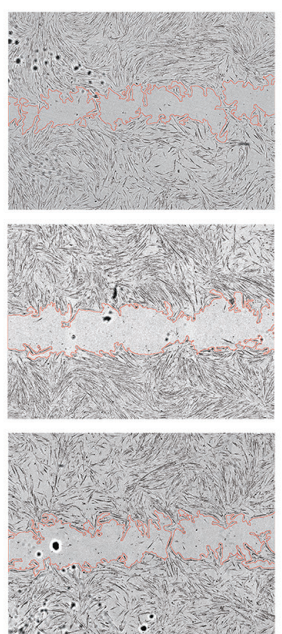

$48 \mathrm{~h}$

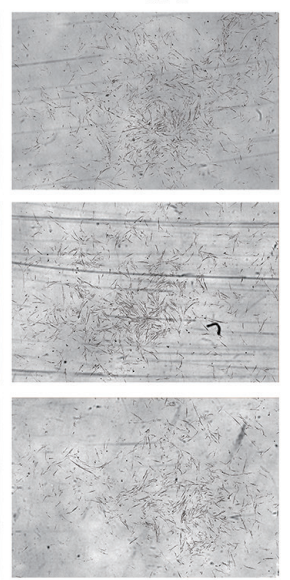

$48 \mathrm{~h}$
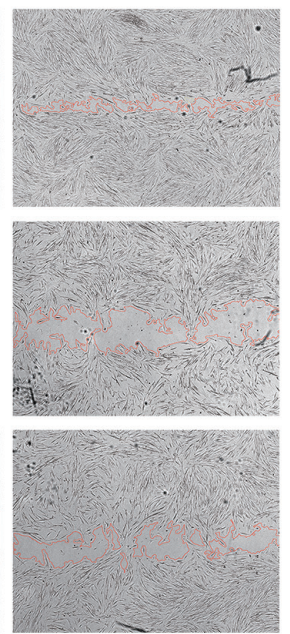
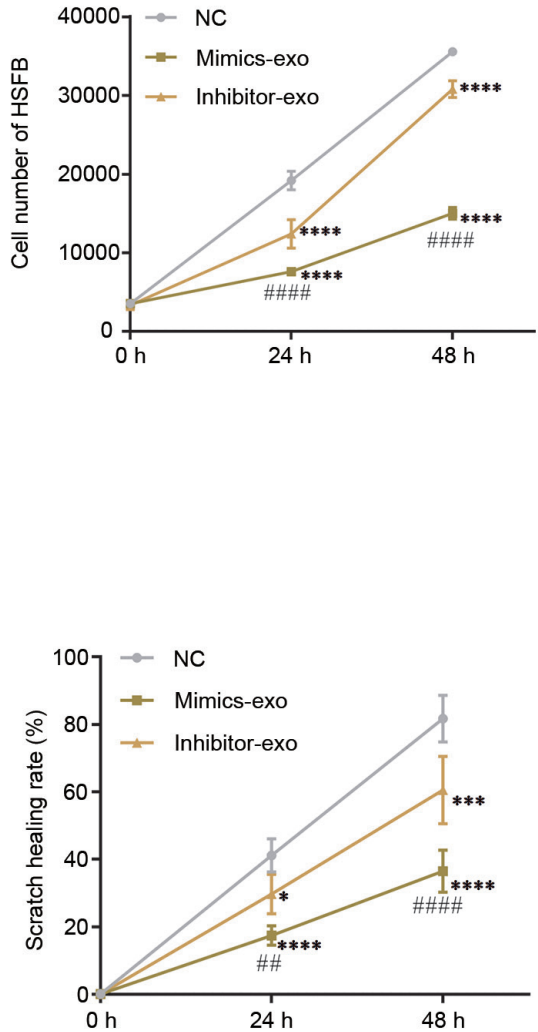

Figure 3. Effect of miR-29a-modified hADSCs-exo on the proliferation and migration of HSFBs. (A) Proliferation of HSFBs treated with different hADSCs-exo. (B) Effect of miR-29a-modified hADSCs-exo on the scratch healing of HSFBs (magnification, x10). Two-way ANOVA followed by Bonferroni post hoc test was used

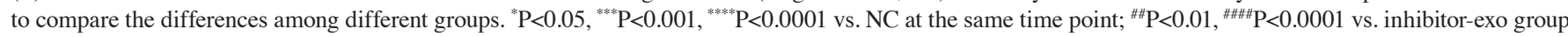
at the same time point. NC, negative control; mimics-exo, exosomes from miR-29a overexpressed hADSCs treated group; inhibitor-exo, exosomes from miR-29a knockdown hADSCs treated group; miR, microRNA; hADSCs, human adipose-derived mesenchymal stem cells; HSFBs, human hypertrophic scar fibroblasts.

average particle size of exosomes was $81.14 \mathrm{~nm}$ and $96.62 \%$ of the total particles were $30-150 \mathrm{~nm}$ in diameter, and the average concentration of exosomes was $1.43 \mathrm{E}+10 / \mathrm{ml}$ (Fig. 2B).

Next, the biomarkers of exosomes, including CD63, CD81 and TSG101, and the cellular protein actin were detected via western blotting. The results demonstrated that the expression levels of CD63, CD81 and TSG101 were low in hADSCs, but were high in hADSCs-exo, while the expression level of the cellular protein actin was low in hADSCs-exo (Fig. 2C). Additionally, nanoflow cytometry revealed that the positive rates of the exosome membrane proteins CD63 and CD81 were both $>30 \%$ (Fig. 2D), which indicated that the exosomes were successfully extracted from hADSC cultures.

Exosomes derived from miR-29a-overexpressing and miR-29a-inhibited hADSCs were added to the culture medium of HSFBs, and were labeled with an anti-PKH67 antibody, and DAPI was used to label the nuclei of HSFBs to determine whether HSFBs can directly take up hADSCs-exo. The results indicated that HSFBs can directly take up hADSCs-exo (Fig. 2E). Then, the expression levels of miR-29a in different groups of hADSCs-exo and HSFBs were detected via RT-qPCR. First, it was found that miR-29a mimics and inhibitors could effectively regulate the content of miR-29a in hADSCs-exo after hADSCs were transfected with miR-29a mimics or inhibitor (Fig. 2F). In addition, compared with the untreated hADSCs-exo-treated group, the mimics-exo-treated group showed higher expression of miR-29a, while the inhibitor-exo-treated group showed lower expression of miR-29a (Fig. 2G). These results suggested that miR-29a-modified hADSCs-exo can regulate the expression of miR-29a in HSFBs.

Effect of miR-29a-modified hADSCs-exo on the proliferation and migration of HSFBs. To evaluate the effect of miR-29a-modified hADSCs-exo on HSFBs, HSFBs were divided into different groups and treated with mimics-exo or inhibitor-exo. PBS was added as a NC, and then the proliferation of HSFBs was imaged and observed. As shown in Fig. 3A, HSFBs treated with mimics-exo showed a lower proliferative ability, while the proliferative rates of HSFBs in the NC and inhibitor-exo-treated groups were higher. Furthermore, a cell scratch healing assay was performed to determine the influence of miR-29a-modified hADSCs-exo on HSFB migration. Compared with the NC group, miR-29a-overexpressing 
A

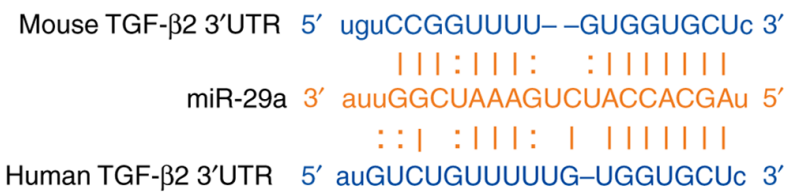

C

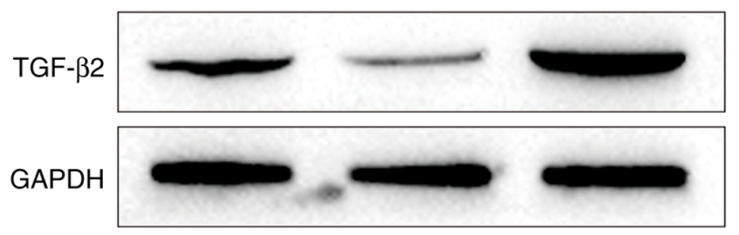

B
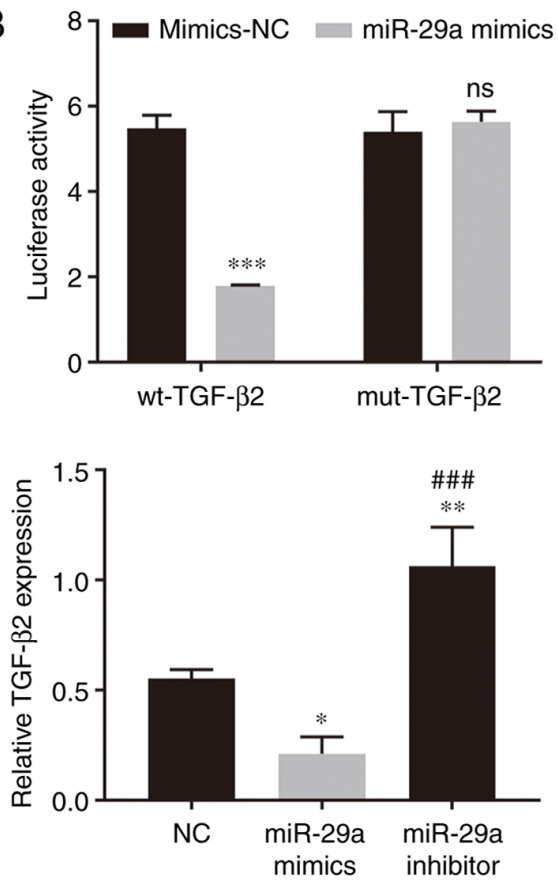

Figure 4. Relationship between TGF- $\beta 2$ and miR-29a. (A) The binding site between miR-29a and the 3 'UTR of TGF- $\beta 2$ mRNA was predicted by the StarBase dataset. (B) Dual-luciferase reporter assay results indicated that miR-29a can directly bind to the TGF- 32 3'UTR. Two-way ANOVA followed by Bonferroni post hoc test was used to compare the differences among groups. ${ }^{* * * *} \mathrm{P}<0.001$ vs. mimics-NC group. (C) Effect of miR-29a overexpression and knockdown on the expression level of TGF- 32 in HSFBs. One-way ANOVA followed by Bonferroni post hoc test was used to compare the differences among different groups. ${ }^{*} \mathrm{P}<0.05$, ${ }^{* *} \mathrm{P}<0.01 \mathrm{vs.} \mathrm{NC}$ group; ${ }^{\# \#} \mathrm{P}<0.001$ vs. miR-29a mimics group. miR, microRNA; wt, wild-type; mut, mutant; UTR, untranslated region; NC, negative control; ns, not significant.

hADSCs-exo significantly inhibited the migration of HSFBs in the mimics-exo-treated group, while the migration of HSFBs in the inhibitor-exo-treated group was enhanced compared with that in the mimics-exo-treated group (Fig. 3B). These results indicated that hADSC-exo-derived miR-29a can inhibit the proliferation and migration of HSFBs.

miR-29a-modified hADSCs-exo regulates the TGF- $\beta 2 /$ Smad3 signaling pathway in HSFBs. To verify whether hADSC-exo-derived miR-29a inhibits HSFB proliferation and migration via the TGF- $\beta /$ Smad signaling pathway, the targets of miR-29a were scanned in the StarBase online database, and TGF- $\beta 2$ was predicted to be one of the targets of miR-29a. There was a binding site between miR-29a and the 3'UTR of TGF- $\beta 2$ mRNA (Fig. 4A). Further results from the dual-luciferase reporter assay revealed that miR-29a mimics inhibited the luciferase activity of the wild-type TGF- $\beta 2$ 3'UTR (Fig. 4B), which indicated that miR-29a can directly bind to the TGF- $\beta 2$ 3'UTR. The western blotting results demonstrated that miR-29a mimic transfection significantly inhibited the expression level of TGF- $\beta 2$ in HSFBs, while miR-29a inhibitor transfection upregulated TGF- $\beta 2$ expression in HSFBs (Fig. 4C). These results suggested that miR-29a can inhibit the expression of TGF- $\beta 2$ by binding to the 3'UTR of TGF- $\beta 2$ mRNA.

To test whether miR-29a-modified hADSCs-exo inhibit collagen deposition and the fibrosis of HSFBs via the TGF- $\beta 2 / \mathrm{Smad} 3$ signaling pathway, the TGF- $\beta 2 / \mathrm{Smad} 3$ agonist SRI-011381 hydrochloride $(10 \mu \mathrm{M})$ and the TGF- $\beta$ signaling inhibitor pirfenidone (AMR69; $3 \mathrm{mM}$ ) were used to co-treat HSFBs with mimics-exo and inhibitor-exo, respectively. Western blot analysis was performed to evaluate the expression levels of TGF- $\beta 2$, p-Smad3, Col-I, Col-III and the fibrotic gene $\alpha$-SMA in different groups of HSFBs. The data demonstrated that mimics-exo significantly inhibited the expression levels of TGF- $\beta 2$ and p-Smad3 (Fig. 5A-C), as well as downregulated the expression levels of Col-I, Col-III and $\alpha$-SMA(Fig.5A and D-F), whileSRI-011381 reversed the inhibition of miR-29a-overexpressing hADSCs-exo on the activation of TGF- $32 / \mathrm{Smad} 3$ signaling and the fibrosis of HSFBs (Fig. 5). Moreover, AMR69 and inhibitor-exo cotreatment enhanced the expression level of p-Smad3 (Fig. 5A-C) and upregulated the expression levels of Col-I and Col-III and compared with the mimics-exo-treated group (Fig. 5A and D-F), whereas there was no significant difference in the expression of TGF- $\beta 2$ and $\alpha$-SMA between the AMR69/inhibitor-exo cotreatment and mimics-exo-treated groups. Thus, inhibition of the TGF- $\beta 2 / \mathrm{Smad} 3$ signaling pathway may be a potential mechanism via which miR-29a-modified hADSCs-exo reduce excessive scar formation during skin wound healing.

Effects of miR-29a-overexpressing hADSCs-exo on scar formation in scalded mice. To evaluate the inhibitory effect of miR-29a-overexpressing hADSCs-exo on scar formation, a scalded skin mouse model was established, and miR-29a mimics-exo was subcutaneously injected around the wound. From the gross observation of scar formation and wound healing after thermal injury, miR-29a-overexpressing hADSCs-exo showed an obvious effect on promoting the healing of thermal injury, and SRI-011381 reversed the effect of miR-29a-overexpressing hADSCs-exo (Fig. 6A and B). As shown in Fig. 6C, the expression level of miR-29a was significantly downregulated in the thermally injured group. Subcutaneous injection of miR-29a-overexpressing hADSCs-exo led to an upregulation 

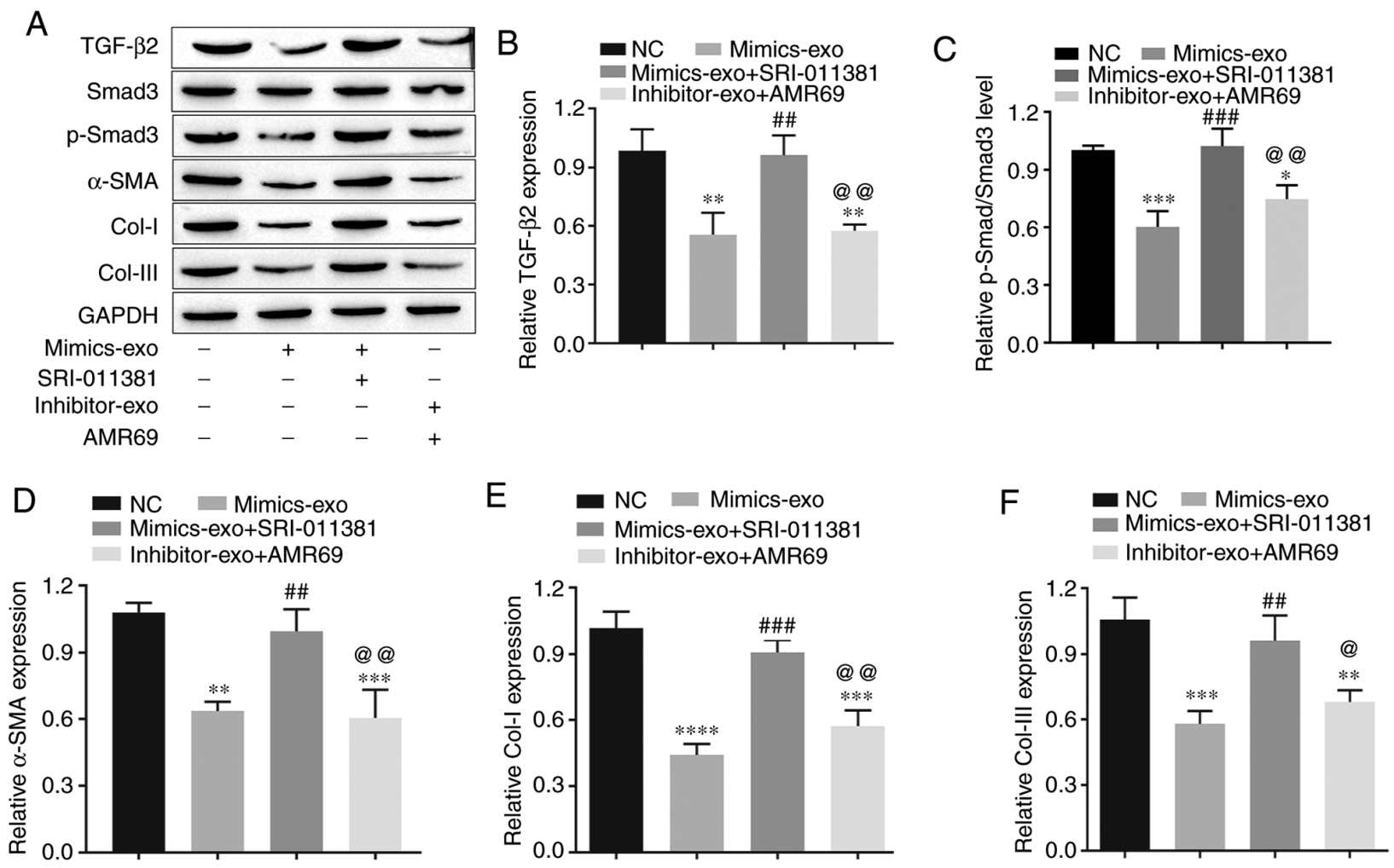

Figure 5. Effects of miR-29a-modified hADSCs-exo on the TGF- $\beta 2 / S m a d 3$ signaling pathway and fibrosis of human hypertrophic scar fibroblasts. (A) Western blotting results. (B) Relative expression level of TGF- $\beta 2$. (C) Relative expression level of p-Smad3. (D) Relative expression level of the fibrosis gene $\alpha$-SMA. (E) Relative expression level of Col-I. (F) Relative expression level of Col-III. One-way ANOVA followed by Bonferroni post hoc test was used to compare the differences among different groups. ${ }^{~} \mathrm{P}<0.05,{ }^{* *} \mathrm{P}<0.01,{ }^{* * *} \mathrm{P}<0.001,{ }^{* * * *} \mathrm{P}<0.0001$ vs. $\mathrm{NC}$ group; ${ }^{\# \#} \mathrm{P}<0.01,{ }^{\# \# *} \mathrm{P}<0.001$ vs. mimics-exo group; ${ }^{\circledR} \mathrm{P}<0.05,{ }^{@}{ }^{@} \mathrm{P}<0.01$ vs. mimics-exo + SRI-011381 group. Col-I, collagen I; Col-III, collagen III; miR, microRNA; NC, negative control; $\alpha$-SMA, $\alpha$-smooth muscle actin; p-, phosphorylated; mimics-exo, exosomes from miR-29a overexpressed hADSCs treated group; inhibitor-exo, exosomes from miR-29a knockdown hADSCs treated group; hADSCs, human adipose-derived mesenchymal stem cells.

of miR-29a, and the agonist of TGF- $\beta$, SRI-011381, had no obvious effect on the expression level of miR-29a in the skin tissues.

Next, histopathological morphological changes in scalded tissue were determined via H\&E staining. Thick dermis and disordered granulation tissue, obvious infiltration of inflammatory cells, dense collagen fibers and irregular arrangement of collagen bundles were observed in thermally injured tissues. Compared with the thermal injury group, miR-29a-overexpressing hADSCs-exo markedly reduced the aforementioned morphological changes, while SRI-011381 reversed the inhibitory effect of mimics-exo on scar formation (Fig. 6D). Furthermore, Fig. 6E shows the epidermal thickness measurement results in each group, suggesting that miR-29a-overexpressing hADSCs-exo could inhibit epidermal overgrowth during skin wound repair.

Masson staining was performed to observe the fibrosis of the tissues (Fig. 6F and G). The dermis of thermally injured tissue was mainly composed of irregularly dense and disordered blue-stained collagen fibers, while the mimics-exo treatment group showed more regular loose collagen fibers. Moreover, the deposition of collagen fibers in the SRI-011381 treatment group was similar to that in the thermal injury group.

Effect of miR-29a-overexpressing hADSCs-exo on the TGF- $\beta 2 /$ Smad3 signaling pathway in scar tissues. To further verify the molecular mechanism by which hADSC-exo-derived
miR-29a inhibits scar formation via the TGF- $\beta 2 / \operatorname{Smad} 3$ signaling pathway, the protein expression levels of TGF- $\beta 2$, p-Smad3, the fibrosis gene $\alpha$-SMA and two major representative collagens, Col-I and Col-III, were measured in different groups of scar tissues. As shown in Fig. 7A-C, compared with the control group, the TGF- $\beta 2 / \mathrm{Smad} 3$ signaling pathway was markedly activated in thermally injured tissues, and mimics-exo treatment inhibited the activation of TGF- $\beta 2 / \mathrm{Smad} 3$ signaling, while SRI-011381 reversed the inhibition of mimics-exo on this pathway. Furthermore, the expression levels of $\alpha$-SMA, Col-I and Col-III were significantly inhibited by miR-29a-overexpressing hADSCs-exo compared with the thermal injury group, but the agonist of TGF- $\beta 2 / \mathrm{Smad} 3$, SRI-011381, reduced this inhibition (Fig. 7A and D-F). These results indicated that miR-29a-overexpressing hADSCs-exo inhibited excessive scar formation by inhibiting the activation of the TGF- $\beta 2 / \mathrm{Smad} 3$ signaling pathway.

\section{Discussion}

During wound healing, scar formation and hyperplasia often occur, not only affecting aesthetics but also causing pain, itching and other symptoms. These scars and hyperplasia may also have negative impacts on the patient's physical, psychological and social functions, and have become an urgent problem to be solved in the field of plastic surgery (4). Hypertrophic scars or keloids are more common types of pathological 

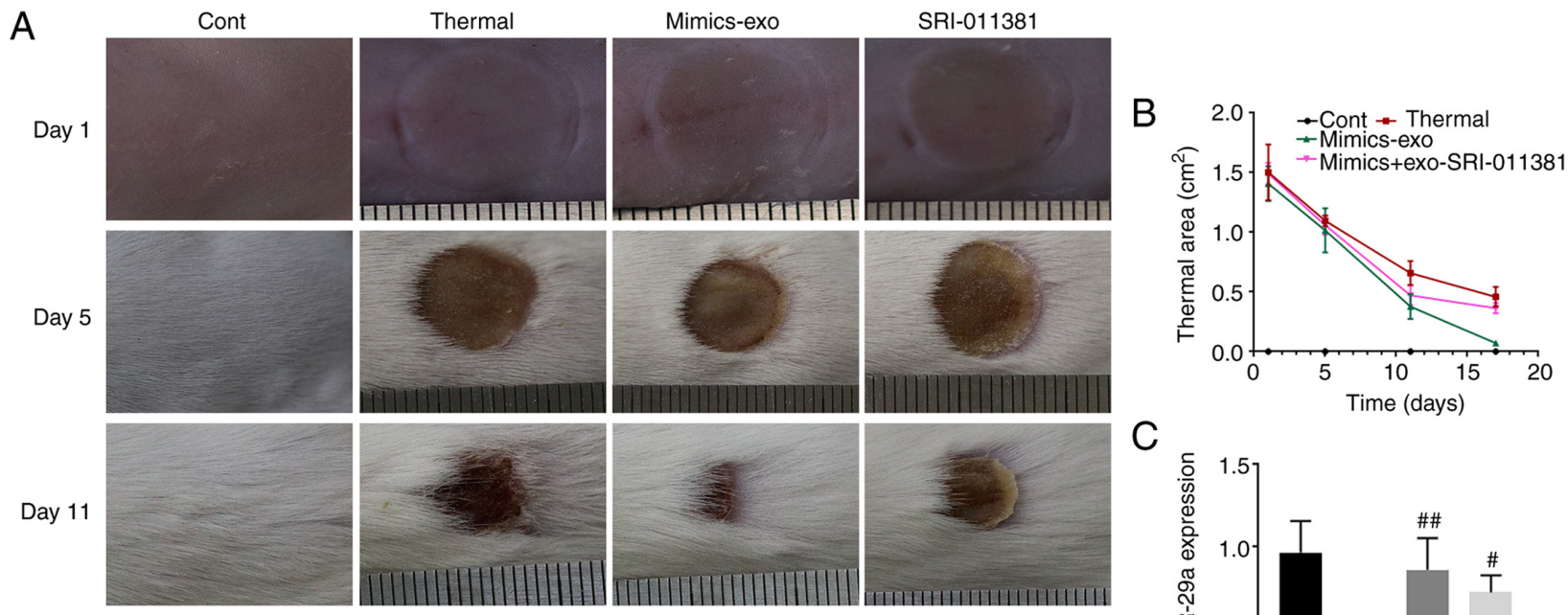

C
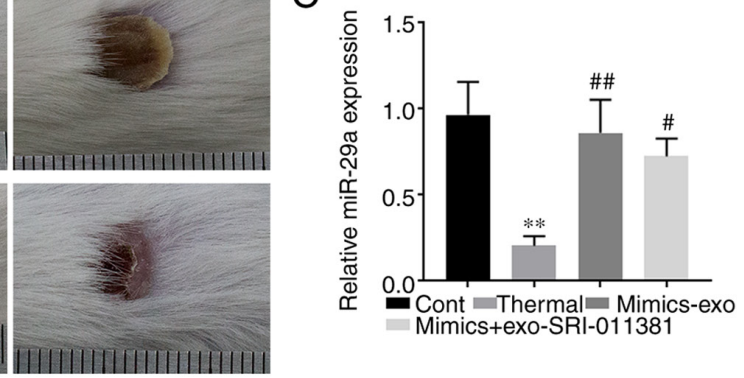

D

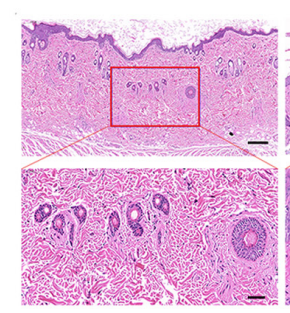

Cont

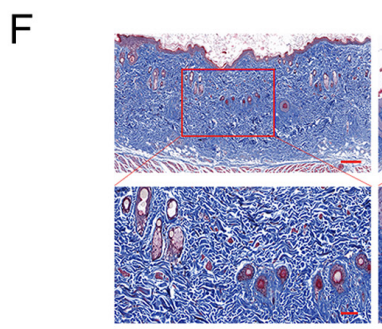

Cont

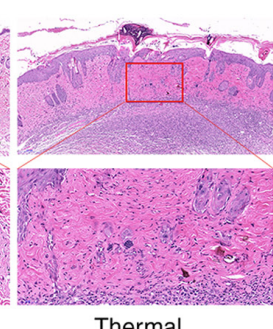

Thermal

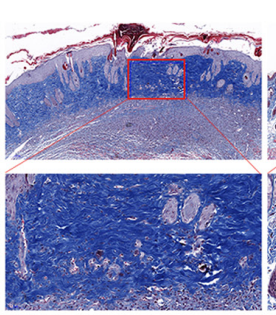

Thermal

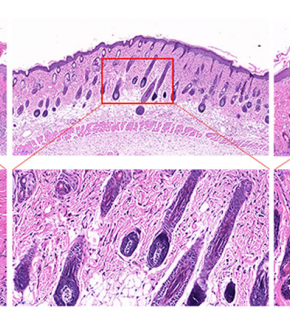

Mimics-exo

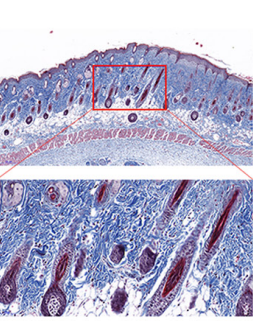

Mimics-exo
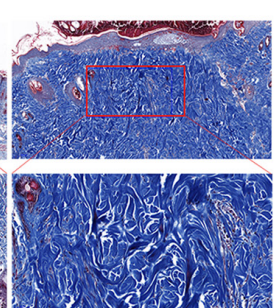

SRI-011381
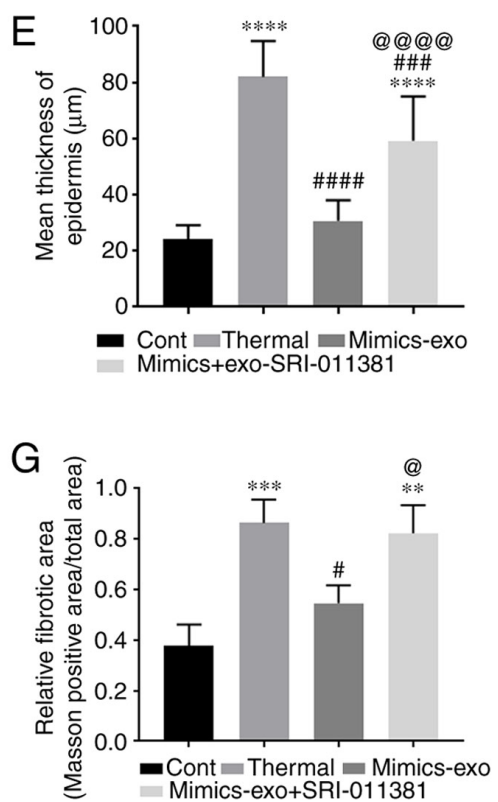

Figure 6. Effect of miR-29a-overexpressing hADSCs-exo on scar formation in thermally injured mice. (A) Representative images of thermal injury of mice on days 1, 5, 11 and 17 after injury. (B) Thermal areas in each group. (C) miR-29a expression in thermally injured tissues of different groups. (D) Representative images of H\&E staining of scar tissues. (E) Epidermal thicknesses in different groups. (F) Representative images of Masson staining of scar tissues. (G) Relative fibrotic areas in different groups. Bars in the upper panel of D and F indicate $200 \mu \mathrm{m}$, while the bar indicates $50 \mu \mathrm{m}$ in the lower panel. One-way ANOVA followed by Bonferroni post hoc test was used to compare the differences among different groups. ${ }^{* *} \mathrm{P}<0.01,{ }^{* * * *} \mathrm{P}<0.001,{ }^{* * * * *} \mathrm{P}<0.0001$ vs. Cont group; ${ }^{\#} \mathrm{P}<0.05,{ }^{\#} \mathrm{P}<0.01,{ }^{\# \#} \mathrm{P}<0.001,{ }^{\# \# \# \#} \mathrm{P}<0.0001$ vs. thermal group; ${ }^{\circledR} \mathrm{P}<0.05$, ${ }^{\circledR} @ @ \mathrm{P}<0.0001$ vs. mimics-exo group. Cont, control; mimics-exo, exosomes from miR-29a overexpressed hADSCs treated group; inhibitor-exo, exosomes from miR-29a knockdown hADSCs treated group; hADSCs, human adipose-derived mesenchymal stem cells; miR, microRNA.

scars in clinical practice, and their incidence is increasing, with $\sim 11$ million patients with keloids in high-income countries in 2000 (40). The excessive proliferation and fibrosis of fibroblasts caused by the activation of the TGF- $\beta / \mathrm{Smad}$ signaling pathway contribute primarily to the formation of pathological scars $(2,32)$. The present study demonstrated that miR-29a-modified hADSCs-exo inhibited the proliferation and migration of HSFBs and reduced excessive scar formation via the targeted inhibition of the TGF- $\beta 2 / \mathrm{Smad} 3$ signaling pathway.
Several studies have reported that only a small part of the tissue repair function of stem cells involves the proliferation and differentiation of stem cells in the damaged area, while most of them function via paracrine signaling (41). Exosomes reflect not only the physiological state of the source cells, but also the differentiation direction of secreted cells. The miRNAs contained in exosomes are an important medium for intercellular communication; after exosomes enter target cells, they are regulated by degradation and re-expression and alter the genes of target cells (13). The expression profile of RNA in 
A
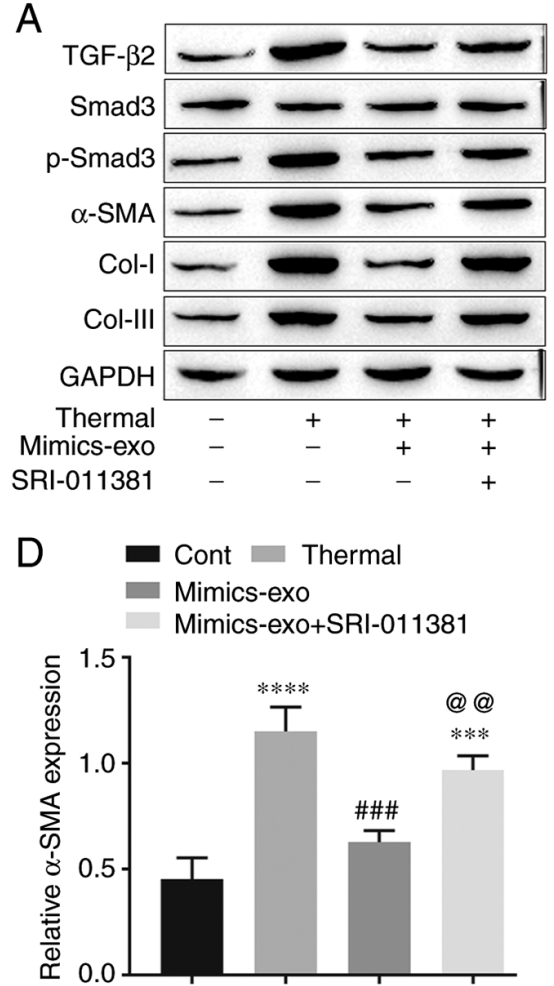

B
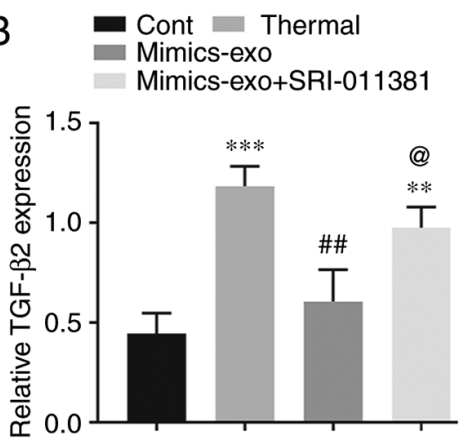

E

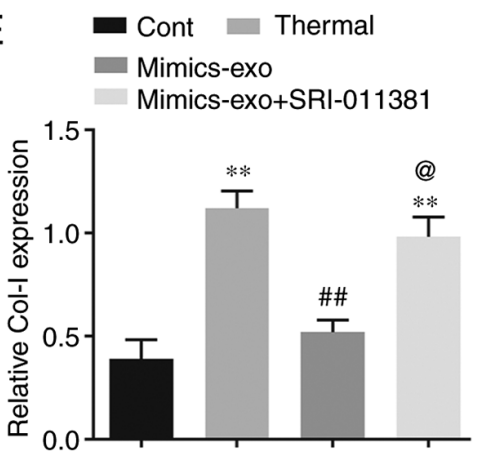

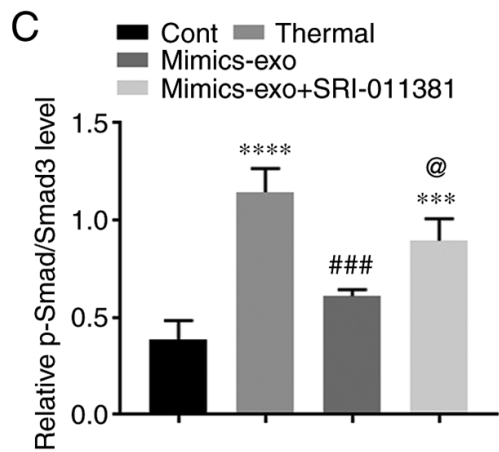

$\mathrm{F}$

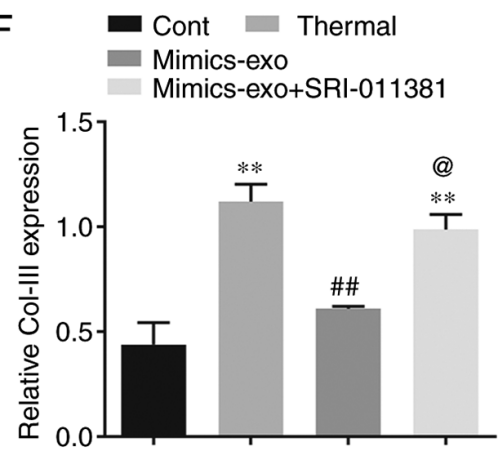

Figure 7. Effect of microRNA-29a-modified hADSCs-exo on the TGF- $\beta 2 /$ Smad3 signaling pathway in scar tissues. (A) Western blotting results. (B) Relative expression level of TGF- $\beta 2$. (C) Relative expression level of p-Smad3. (D) Relative expression level of the fibrosis gene $\alpha$-SMA. (E) Relative expression level of Col-I. (F) Relative expression level of Col-III. One-way ANOVA followed by Bonferroni post hoc test was used to compare the differences among different groups. ${ }^{* *} \mathrm{P}<0.01,{ }^{* * * *} \mathrm{P}<0.001,{ }^{* * * *} \mathrm{P}<0.0001$ vs. Cont group; ${ }^{\# \#} \mathrm{P}<0.01,{ }^{\# \# \#} \mathrm{P}<0.001$ vs. thermal group; ${ }^{\circledR} \mathrm{P}<0.05$, ${ }^{@} @ \mathrm{P}<0.01$ vs. mimics-exo group. Col-I, collagen I; Col-III, collagen III; Cont, control; $\alpha$-SMA, $\alpha$-smooth muscle actin; p-, phosphorylated; mimics-exo, exosomes from miR-29a overexpressed hADSCs treated group; hADSCs, humanadipose-derived mesenchymal stem cells.

cells not only reflects donor specificity, but the RNA contained in exosomes is affected by the type of differentiated cells; thus, exosomes from different cell sources express different biological characteristics (42). Previous studies have also confirmed that MSC-derived exosomes, including adipose stem cells and umbilical cord stem cells, have the ability to promote wound healing in animal models $(43,44)$. These functions mainly depend on miRNAs carried by exosomes, such as miR-31 and miR-125a, as stimulation signals, which promote the migration, proliferation and angiogenesis of endothelial cells $(45,46)$. It has also been revealed that exosomes can reduce scar formation and the proliferation of myofibroblasts in a mouse model of skin defects. High-throughput sequencing and functional analysis have demonstrated that exosomes carrying miR-21, miR-23a, miR-125b and miR-145 inhibit the TGF- $\beta 2 / \mathrm{Smad} 2$ signaling pathway, thereby inhibiting the formation of myofibroblasts and preventing scar formation (47). The current study revealed that hADSCs-exo inhibited pathological scar formation in scalded mice by transmitting exogenous miR-29a, thus reducing collagen deposition in tissues.

TGF- $\beta$ serves an important role and mediates a complex mechanism in the process of scar formation. It participates in the regulation of a variety of biological substances and response processes, including extracellular matrix and proteases, as well as the proliferation, differentiation and migration of various cells (47). Currently, it is considered that during scar formation, TGF- $\beta$ can regulate the activation of downstream Smad signaling pathways $(48,49)$. Moreover, any abnormal expression of the TGF- $\beta /$ Smad signaling pathway may cause scar hyperplasia. A variety of molecules and proteins have been shown to promote or inhibit the transmission of signals, such as Toll-like receptors, miRNAs, TNF receptor-related TNF receptor-associated protein 1-like protein and Smad interacting protein 1 . The abnormal expression of miRNAs during fibrosis can serve a promotive or inhibitory role by participating in various processes in inflammatory pathways or the immune response. For example, miRNAs can promote or inhibit various links in the TGF- $\beta /$ Smad signaling pathway to promote or inhibit scar formation (49). The results of the present study also indicated that miR-29a-modified hADSCs-exo therapy can inhibit the TGF- $\beta 2 /$ Smad3 signaling pathway via its derived miR-29a to inhibit collagen deposition and extracellular matrix fibrosis to further inhibit scar formation.

In conclusion, the present study provided evidence that miR-29a-overexpressing hADSC-derived exosomes can inhibit the TGF- $\beta 2 / \mathrm{Smad} 3$ signaling pathway in wound tissues to promote wound healing and reduce pathological scar formation by transmitting exogenous miR-29a to wound tissues. These results suggested that miR-29a-modified hADSCs-exo may be a potential therapeutic for skin wound healing and pathological scars.

\section{Acknowledgements}

Not applicable. 


\section{Funding}

This study was supported by grants from the Yunnan Science and Technology Department-Kunming Medical University Joint Fund [grant no. 2017FE467(-051)].

\section{Availability of data and materials}

The datasets used and/or analyzed during the current study are available from the corresponding author upon reasonable request.

\section{Authors' contributions}

All authors contributed substantially to this manuscript. RY contributed primarily to this manuscript by participating in all the experiments and writing the manuscript. XD participated primarily in the in vivo testing. YL contributed mainly to the in vitro testing. CL contributed to the data collation and analysis. LL contributed to the conception and design of this study, and guided the writing of the manuscript. RY and LL confirmed the authenticity of the raw data. All authors read and approved the final manuscript.

\section{Ethics approval and consent for publication}

All experimental mouse protocols were approved by the Animal Ethics Committee of The First Affiliated Hospital of Kunming Medical University (approval no. kmmu2021188), and the animals were handled according to the management requirements of the Animal Management Association of The First Affiliated Hospital of Kunming Medical University.

\section{Patient consent for publication}

Not applicable.

\section{Competing interests}

The authors declare that they have no competing interests.

\section{References}

1. Schultz G, Davidson J, Kirsner R, Bornstein P and Herman I: Dynamic reciprocity in the wound microenvironment. Wound Repair Regen 19: 134-148, 2011.

2. Karppinen S, Heljasvaara R, Gullberg D, Tasanen K and Pihlajaniemi T: Toward understanding scarless skin wound healing and pathological scarring. F1000Res 8: F1000, 2019.

3. Brown BC, Moss TP, McGrouther DA and Bayat A: Skin scar preconceptions must be challenged: Importance of self-perception in skin scarring. J Plast Reconstr Aesthet Surg 63: 1022-1029, 2010.

4. van den Broek LJ, Limandjaja GC, Niessen FB and Gibbs S: Human hypertrophic and keloid scar models: Principles, limitations and future challenges from a tissue engineering perspective. Exp Dermatol 23: 382-386, 2014.

5. Yu J, Wang MY, Tai HC and Cheng NC: Cell sheet composed of adipose-derived stem cells demonstrates enhanced skin wound healing with reduced scar formation. Acta Biomater 77: 191-200, 2018.

6. Liu SC, Bamodu OA, Kuo KT, Fong IH, Lin CC, Yeh CT and Chen SG: Adipose-derived stem cell induced-tissue repair or wound healing is mediated by the concomitant upregulation of miR-21 and miR-29b expression and activation of the AKT signaling pathway. Arch Biochem Biophys 705: 108895, 2021.
7. Ni X, Shan X, Xu L, Yu W, Zhang M, Lei C, Xu N, Lin J and Wang B: Adipose-derived stem cells combined with platelet-rich plasma enhance wound healing in a rat model of full-thickness skin defects. Stem Cell Res Ther 12: 226, 2021.

8. Cai Y, Li J, Jia C, He Y and Deng C: Therapeutic applications of adipose cell-free derivatives: A review. Stem Cell Res Ther 11: 312, 2020.

9. Basu J and Ludlow JW: Exosomes for repair, regeneration and rejuvenation. Expert Opin Biol Ther 16: 489-506, 2016.

10. Merino-GonzálezC,ZuñigaFA,Escudero C, Ormazabal V, Reyes C, Nova-Lamperti E, Salomón C and Aguayo C: Mesenchymal stem cell-derived extracellular vesicles promote angiogenesis: Potencial clinical application. Front Physiol 7: 24, 2016.

11. Konala VB, Mamidi MK, Bhonde R, Das AK, Pochampally R and Pal R: The current landscape of the mesenchymal stromal cell secretome: A new paradigm for cell-free regeneration. Cytotherapy 18: 13-24, 2016.

12. Casado-Díaz A, Quesada-Gómez JM and Dorado G: Extracellular vesicles derived from mesenchymal stem cells (MSC) in regenerative medicine: Applications in skin wound Healing. Front Bioeng Biotechnol 8: 146, 2020.

13. Rani S and Ritter T: The exosome - A naturally secreted nanoparticle and its application to wound healing. Advanced materials (Deerfield Beach, Fla.) 28: 5542-5552, 2016.

14. Rani S, Ryan A, Griffin M and Ritter T: Mesenchymal stem cell-derived extracellular vesicles: Toward cell-free therapeutic applications. Molecular therapy : the journal of the American Society of Gene Therapy 23: 812-823, 2015.

15. Shabbir A, Cox A, Rodriguez-Menocal L, Salgado M and Van Badiavas E: Mesenchymal stem cell exosomes induce proliferation and migration of normal and chronic wound fibroblasts, and enhance angiogenesis in vitro. Stem Cells Dev 24: 1635-1647, 2015.

16. Diegelmann R and Evans M: Wound healing: an overview of acute, fibrotic and delayed healing. Front Biosci 9: 283-289, 2004.

17. Yuan H, Guan J, Zhang J, Zhang R and Li M: Exosomes secreted by human urine-derived stem cells accelerate skin wound healing by promoting angiogenesis in rat. Cell Biol Int 41: 933, 2017.

18. Hu L, Wang J, Zhou X, Xiong Z, Zhao J, Yu R, Huang F, Zhang H and Chen L: Exosomes derived from human adipose mensenchymal stem cells accelerates cutaneous wound healing via optimizing the characteristics of fibroblasts. Sci Rep 6: 32993, 2016.

19. Li X, Liu L, Yang J, Yu Y, Chai J, Wang L, Ma L and Yin H: Exosome derived from human umbilical cord mesenchymal stem cell mediates MiR-181c attenuating burn-induced excessive inflammation. EBioMedicine 8: 72-82, 2016.

20. Tafrihi M and Hasheminasab E: MiRNAs: Biology, biogenesis, their Web-based tools, and databases. MicroRNA 8: 4-27, 2019.

21. Ha M and Kim VN: Regulation of microRNA biogenesis. Nat Rev Mol Cell Biol 15: 509-524, 2014.

22. Dietrich C, Singh M, Kumar N and Singh SR: The emerging roles of microRNAs in stem cell aging. Adv Exp Med Biol 1056: $11-26,2018$

23. Cheng J, Wang Y, Wang D and Wu Y: Identification of collagen 1 as a post-transcriptional target of miR-29b in skin fibroblasts: Therapeutic implication for scar reduction. Am J Med Sci 346: 98-103, 2013

24. Murali VP and Holmes CA: Mesenchymal stromal cell-derived extracellular vesicles for bone regeneration therapy. Bone Rep 14: 101093, 2021

25. Ichinohe N, Ishii M, Tanimizu N, Mizuguchi T, Yoshioka Y, Ochiya T, Suzuki H and Mitaka T: Extracellular vesicles containing miR-146a-5p secreted by bone marrow mesenchymal cells activate hepatocytic progenitors in regenerating rat livers. Stem Cell Res Ther 12: 312, 2021.

26. Du Y and Ning JZ: MiR-182 Promotes ischemia/reperfusioninduced acute kidney injury in rat by targeting FoxO3. Urol Int 105: 687-696, 2021.

27. Ouyang $\mathrm{Z}$ and Wei K: miRNA in cardiac development and regeneration. Cell Regen (Lond) 10: 14, 2021.

28. Zhou J, Zhang X, Liang P, Ren L, Zeng J, Zhang M, Zhang P and Huang X: Protective role of microRNA-29a in denatured dermis and skin fibroblast cells after thermal injury. Biol Open 5: 211-219, 2016.

29. Guo L, Huang X, Liang P, Zhang P, Zhang M, Ren L, Zeng J, Cui X and Huang X: Role of XIST/miR-29a/LIN28A pathway in denatured dermis and human skin fibroblasts (HSFs) after thermal injury. J Cell Biochem 119: 1463-1474, 2018.

30. Zgheib C, Hodges M, Hu J, Beason DP, Soslowsky LJ, Liechty KW and $\mathrm{Xu} \mathrm{J}$ : Mechanisms of mesenchymal stem cell correction of the impaired biomechanical properties of diabetic skin: The role of miR-29a. Wound Repair Regen 24: 237-246, 2016. 
31. Wang Z, Feng C, Song K, Qi Z, Huang W and Wang Y: IncRNA-H19/miR-29a axis affected the viability and apoptosis of keloid fibroblasts through acting upon COL1A1 signaling. J Cell Biochem 121: 4364-4376, 2020.

32. Zhang T, Wang X, Wang Z, Lou D, Fang QQ, Hu YY, Zhao WY, Zhang LY, Wu LH, Tan WQ: Current potential therapeutic strategies targeting the TGF- $\beta /$ Smad signaling pathway to attenuate keloid and hypertrophic scar formation. Biomed Pharmacother 129: 110287, 2020.

33. Guo J, Lin Q, Shao Y, Rong L and Zhang D: miR-29b promotes skin wound healing and reduces excessive scar formation by inhibition of the TGF- $\beta 1 / \mathrm{Smad} / \mathrm{CTGF}$ signaling pathway. Can J Physiol Pharmacol 95: 437-442, 2017.

34. Zhu Y, Li Z, Wang Y, Li L, Wang D, Zhang W, Liu L, Jiang H, Yang $J$ and Cheng J: Overexpression of miR-29b reduces collagen biosynthesis by inhibiting heat shock protein 47 during skin wound healing. Transl Res 178: 38-53.e6, 2016.

35. Zhang SJ, Yun CJ, Liu J, Yao SY, Li Y, Wang M, Wang C, Bai YY and Xue H: MicroRNA-29a attenuates angiotensin-II induced-left ventricular remodeling by inhibiting collagen, TGF- $\beta$ and SMAD2/3 expression. J Geriatr Cardiol 17: 96-104, 2020.

36. Ragni E, Perucca Orfei C, De Luca P, Viganò M, Colombini A, Lugano G, Bollati $V$ and de Girolamo L: miR-22-5p and miR-29a-5p are reliable reference genes for analyzing extracellular vesicle-associated miRNAs in adipose-derived mesenchymal stem cells and are stable under inflammatory priming mimicking osteoarthritis condition. Stem Cell Rev Rep 15: 743-754, 2019.

37. Wang X, Li Z, Cui Y, Cui X, Chen C and Wang Z: Exosomes isolated from bone marrow mesenchymal stem cells exert a protective effect on osteoarthritis via lncRNA LYRM4-AS1GRPR-miR-6515-5p. Front Cell Dev Biol 9: 644380, 2021.

38. Bian W, Meng B, Li X, Wang S, Cao X, Liu N, Yang M, Tang J, Wang Y and Yang X: OA-GL21, a novel bioactive peptide from Odorrana andersonii, accelerated the healing of skin wounds. Biosci Rep: Jun 21, 2018 (Epub ahead of print). doi: 10.1042/ BSR20180215.

39. Livak KJ and Schmittgen TD: Analysis of relative gene expression data using real-time quantitative PCR and the $2(-\Delta \Delta \mathrm{C}(\mathrm{T}))$ method. Methods 25: 402-408, 2001.

40. Bayat A, McGrouther DA and Ferguson MW: Skin scarring. BMJ 326: 88-92, 2003.
41. New SE,Alvarez-Gonzalez C, Vagaska B, Gomez SG,Bulstrode NW, Madrigal A and Ferretti P: A matter of identity - Phenotype and differentiation potential of human somatic stem cells. Stem Cell Res (Amst) 15: 1-13, 2015.

42. Ren K: Exosomes in perspective: A potential surrogate for stem cell therapy. Odontology 107: 271-284, 2019.

43. Lopez-Verrilli MA, Caviedes A, Cabrera A, Sandoval S, Wyneken U and Khoury M: Mesenchymal stem cell-derived exosomes from different sources selectively promote neuritic outgrowth. Neuroscience 320: 129-139, 2016.

44. Baglio SR, Rooijers K, Koppers-Lalic D, Verweij FJ, Pérez Lanzón M, Zini N, Naaijkens B, Perut F, Niessen HW, Baldini N, et al: Human bone marrow- and adipose-mesenchymal stem cells secrete exosomes enriched in distinctive miRNA and tRNA species. Stem Cell Res Ther 6: 127, 2015.

45. Kang T, Jones TM, Naddell C, Bacanamwo M, Calvert JW, Thompson WE, Bond VC, Chen YE and Liu D: Adipose-derived stem cells induce angiogenesis via microvesicle transport of miRNA-31. Stem Cells Transl Med 5: 440-450, 2016.

46. Liang X, Zhang L, Wang S, Han Q and Zhao RC: Exosomes secreted by mesenchymal stem cells promote endothelial cell angiogenesis by transferring miR-125a.J Cell Sci 129: 2182-2189, 2016.

47. Fang S, Xu C, Zhang Y, Xue C, Yang C, Bi H, Qian X, Wu M, $\mathrm{Ji} \mathrm{K}$, Zhao Y, et al: Umbilical cord-derived mesenchymal stem cell-derived exosomal MicroRNAs suppress myofibroblast differentiation by inhibiting the transforming growth factor- $\beta /$ SMAD2 pathway during wound healing. Stem Cells Transl Med 5: 1425-1439, 2016.

48. Valluru M, Staton CA, Reed MW and Brown NJ: Transforming growth factor- $\beta$ and endoglin signaling orchestrate wound healing. Front Physiol 2: 89, 2011.

49. Gras C, Ratuszny D, Hadamitzky C, Zhang H, Blasczyk R and Figueiredo C: miR-145 contributes to hypertrophic scarring of the skin by inducing myofibroblast activity. Mol Med 21: 296-304, 2015

This work is licensed under a Creative Commons Attribution-NonCommercial-NoDerivatives 4.0 International (CC BY-NC-ND 4.0) License. 